\title{
Multi-disciplinary teams are needed to sensitively diagnose autism in Deaf children
}

\author{
By Dr. Jessica K Edwards
}

An ethnographic study of NHS professionals, who diagnose autism in Deaf children, finds that recognizing the intersections between mental health and Deaf culture is essential for healthcare professionals to make sensitive diagnoses. The study, conducted by researchers in the UK and the Netherlands and published in the journal of Culture, Medicine and Psychiatry was based on an analysis of the practices and perceptions of 16 health service professionals who have conducted autism assessments for Deaf children aged between 0 and 18 years.

Natassia Brenman and colleagues learnt that professionals working in specialist Deaf Services, or with experience working with the Deaf community, had an intersectional understanding of autism assessments. As such, these professionals used their knowledge of how cultural, linguistic, sensory, and social factors interact when making their diagnoses. Many professionals highlighted that Deaf language and culture can be markedly different from social communication observed in the hearing population. Consequently, a commonly vocalized frustration was the emphasis on using a diagnostic system that focuses on comparisons to 'norms' based on populations from a hearing culture.

Undertaking an autism assessment is more complex when the affected child is Deaf. Indeed, the primary issue raised by the professionals involved in this study was how to differentiate between the features or behaviours they should attribute to autism, and which to deafness. Going forward, Brenman and colleagues posit that multi-disciplinary diagnostic teams are necessary to help recognize the various dimensions of a Deaf child's experience and abilities.

\section{Referring to:}

Brenman, N.F., Hiddinga, A. \& Wright, B. (2017), Intersecting cultures in deaf mental health: an ethnographic study of NHS professionals diagnosing autism in D/deaf children. Cult. Med. Psychiatry. 41: 431-452. doi: 10.1007/s11013-0179526-y.

\section{Glossary:}

Intersectionality: a theory that suggests that a person who has numerous identities will have different life experiences (cultural, social and clinical) than a person who shares only one or some of those identities. 